\title{
A Pulsed Triple Ionization Source for Sequential Ion/Ion Reactions in an Electrodynamic Ion Trap
}

\author{
Xiaorong Liang, Hongling Han, Yu Xia, and Scott A. McLuckey \\ Department of Chemistry, Purdue University, West Lafayette, Indiana, USA
}

A pulsed triple ionization source, using a common atmosphere/vacuum interface and ion path, has been developed to generate different types of ions for sequential ion/ion reaction experiments in a linear ion trap-based tandem mass spectrometer. The triple ionization source typically consists of a nano-electrospray emitter for analyte formation and two other emitters, an electrospray emitter and an atmospheric pressure chemical ionization emitter or a second nano-electrospray emitter for formation of the two different reagent ions. The three emitters are positioned in a parallel fashion close to the sampling orifice of the tandem mass spectrometer. The potentials applied to each emitter are sequentially pulsed so that desired ions are generated separately in time and space. Sequential ion/ion reactions take place after analyte ions of interest and different set of reagent ions are sequentially injected into a linear ion trap, where axial trapping is effected by applying an auxiliary radio frequency voltage to the end lenses. The pulsed triple ionization source allows independent optimization of each emitter and can be readily coupled to any atmospheric pressure ionization interface with no need for instrument modifications, provided the potentials required to transmit the ion polarity of interest can be synchronized with the emitter potentials. Several sequential ion/ion reactions examples are demonstrated to illustrate the analytical usefulness of the triple ionization source in the study of gas-phase ion/ion chemistry. (J Am Soc Mass Spectrom 2007, 18, 369-376) (c) 2007 American Society for Mass Spectrometry

G as-phase ion/ion reactions are being explored and utilized for the analysis of the biomolecules via tandem mass spectrometry [1, 2]. Among the various types of ion/ion reactions, proton-transfer and electron-transfer reactions have been most widely studied. Proton-transfer reactions are particularly useful as means for charge state manipulation of biomolecules. Application of proton-transfer reactions include charge reduction of ions derived from electrospray of protein mixtures [3], charge state reduction of product ions following dissociation for simplification of spectral interpretation [4], ion parking [5], and parallel ion parking [6]. Electron-transfer reactions appear to be a highly useful means for deriving the primary structure information of peptides and proteins [7-11].

Most ion/ion reaction studies involving biomolecules are conducted in one of two ways. The first approach is to mix the oppositely charged reactant ions at or near atmospheric pressure before sampling ions into a mass spectrometer. The second approach is to admit the ions of opposite polarity into an electrody-

Published online November 13, 2006

Address reprint requests to Dr. Scott A. McLuckey, Department of Chemistry, Purdue University, West Lafayette, IN 47907-2084, USA. E-mail: mcluckey@purdue.edu namic ion trap for subsequent mutual storage and reaction. The former approach has employed a spray ionization method as one of the ion sources and either a discharge [12-14], radioactive source [15, 16], or another spray ionizer [17] as the second ion source. In this approach, the ion sources have been operated simultaneously and, in general, continuously. This approach avoids the need for instrument modification to accommodate an additional ion source interface but it is not conducive to $\mathrm{MS}^{\mathrm{n}}$ studies. In the latter approach, electrodynamic ion traps, either a quadrupole ion trap or a linear ion trap (LIT), are used as reaction vessels for ion/ion reactions due to their ability to store oppositelycharged ions simultaneously [18] as well as their ion isolation and $\mathrm{MS}^{\mathrm{n}}$ functionalities [19].

Sequential ion/ion reactions have been found to be useful in a number of applications in the analysis of peptides and proteins. One example is the increase of the absolute charge state of a polypeptide ion, which has been achieved via two sequential ion/ion protontransfer reactions involving charge inversion [20,21]. A second example includes phosphopeptide characterization via sequential proton-transfer charge inversion and electron-transfer ion/ion reactions [22], where the first ion/ion reaction inverts the polarity of phosphopeptide sprayed in negative mode and the second ion/ion 
reaction is used to derive structure information from the charge inverted phosphopeptide. Another example is protein identification via sequential electron-transfer and proton-transfer ion/ion reactions [23], in which an electron-transfer reaction is used to derive structure information and a proton-transfer reaction is used to simplify the product ion spectrum by reducing all the highly charged product ions to doubly and singly charged ions. In all of these studies, the spray ionization source has been used to generate and inject the analyte ions via an end-cap electrode, in the case of threedimensional (3-D) ion traps, or along the axial dimension of a linear ion trap. The reagent ions have been generated by distinct ionization sources before injection into the ion trap. A variety of introduction methods for reagent ions have been used, including injecting via the ring electrode, the opposite side of a LIT, or via a turning quadrupole that directs ions into a 3-D ion trap. Examples of the distinct ionization sources include electron ionization [24], in situ chemical ionization [25], and laser desorption [26] within the ion trap and various external ion sources, such as atmospheric sampling glow discharge ionization (ASGDI) [27-29], chemical ionization [7, 8], and spray ionization [30, 31]. However, adapting one or more additional ionization sources onto an ion trap requires major modifications to the tandem mass spectrometer hardware, since only one ion source interface is usually available in a typical commercial instrument.

Three approaches have been demonstrated to allow admission of ions of opposite polarity via the same ion path to induce a single ion/ion reaction in a LIT. One is the use of sonic spray ionization [32], which simultaneously produces ions of opposite polarity and continuously from a single ion source while alternate potentials are applied to the vacuum/atmosphere interface and ion path for admission of each reactant species into a linear ion trap. A second approach involves the use of alternate pulsing of two spray ionizers [33] for formation and transmission of oppositely charged reagents, which allows for the independent optimization of solvent and ionization conditions for each reactant ion and has greater ionization efficiency afforded by nanoelectrospray ionization (nano-ESI) than does sonic spray ionization. The third approach employs the use of pulsed nano-ESI for the formation of multiply charged ions and pulsed corona discharge atmospheric pressure chemical ionization (APCI) [34] for the formation of singly charged reagent ions. This approach is attractive because it enables both single proton-transfer and electron-transfer ion/ion reactions while it shares the same advantages as the second approach, with the exception of the ability to produce multiply charged reagent ions for multiple proton-transfer ion/ion reaction. All three approaches are appropriate for single stage ion/ion reactions. Maximum flexibility in the application of sequential ion/ion reactions for a given analyte species with two or more different reagent ions, however, requires multiple ionization sources.
This paper describes the application of a triple ionization source to the study of sequential ion/ion reactions. A home-built triple ionization source typically consists of a nano-ESI emitter for analyte formation and two other emitters, an ESI emitter and an APCI emitter or a second nano-ESI emitter for formation of the two different reagent ions. The three emitters are positioned in a parallel fashion close to the sampling orifice of the LIT-based tandem mass spectrometer. Axial trapping is enabled by applying an auxiliary radio frequency to the end lenses of one quadrupole array. Sequentially pulsed high potentials were applied to the three emitters, which sequentially generated the ions of desired polarity for a two-step ion/ion reaction. The flexibility and analytical utility of the triple ionization source are illustrated by several key analytical experiments. The significance of the data lies in the convenience with which sequential ion/ion reactions can be readily implemented on an ion trap instrument with minimal modification required. These ion sources share a common atmosphere/vacuum interface as well as a common ion path into a LIT and are used to generate the analyte ions and different reagent ions independently with individually optimized ion injection efficiency.

\section{Experimental}

\section{Materials}

Methanol, ammonium hydroxide, and glacial acetic acid were purchased from Mallinckrodt (Phillipsburg, NJ). Bovine ubiquitin, bradykinin, and azobenzene were obtained from Sigma-Aldrich (St. Louis, MO) and used without further purification. Perfluoro-1-octanol (PFO) and dendrimers were obtained from SigmaAldrich (Milwaukee, WI). The peptide LKRApYGL$\mathrm{NH}_{2}$ was purchase from AnaSpec (San Jose, CA). Solutions of peptides or proteins were dissolved to $5 \mu \mathrm{M}$ in 48/48/2 (vol/vol/vol) methanol/water/acetic acid for positive nano-ESI or in $48 / 48 / 2$ ( $\mathrm{vol} / \mathrm{vol} / \mathrm{vol}$ ) methanol/water/ammonium hydroxide for negative nanoESI. PFO, with a final concentration of $100 \mu \mathrm{M}$, was subjected to negative ESI from 1\% ammonium hydroxide methanol solution. A positive ESI spray solution of poly(propylenimine) (1,4-diaminobutane (DAB) core) dendrimer (generation 4) with a concentration of 0.5 $\mathrm{mg} / \mathrm{mL}$ was prepared in aqueous 5\% acetic acid. A solution of carboxylate-terminated polyamidoamine (PAMAM) dendrimers (generation 1.5) was prepared in 48/48/2 (vol/vol/vol) methanol/water/ammonium hydroxide with a concentration of $1 \mathrm{mg} / \mathrm{mL}$ for negative electrospray.

\section{Apparatus Setup}

A home-built triple ionization source was positioned directly in front of the atmosphere-vacuum interface of a modified SCIEX 2000 Q TRAP mass spectrometer as shown schematically in Figure 1. 


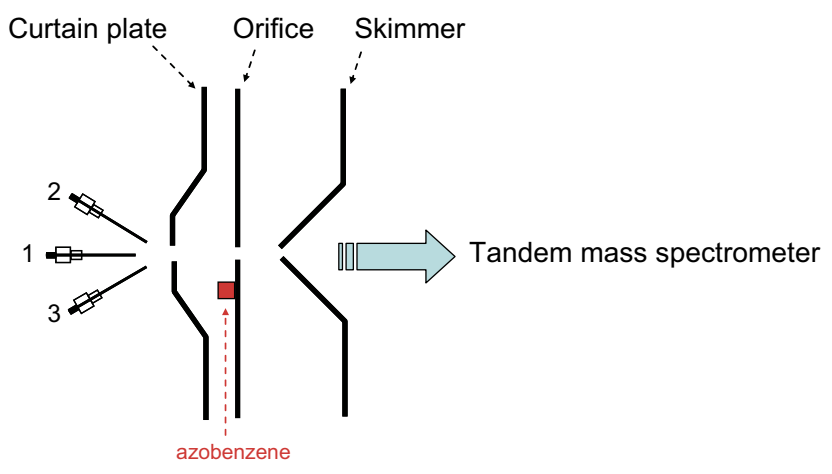

Figure 1. Schematic of a triple ionization source couple with the modified Q TRAP mass spectrometer (only the vacuum/atmosphere interface of the Q TRAP instrument, which was unmodified, is represented in the schematic diagram).

The triple ionization source typically consists of a nano-electrospray emitter for analyte formation and two other emitters, an electrospray emitter and an atmospheric pressure chemical ionization emitter or a second nano-electrospray emitter for formation of the two different reagent ions. The three emitters were clamped separately on a capillary holder and positioned in a parallel fashion with 3 to $5 \mathrm{~mm}$ between the tips and 10 to $15 \mathrm{~mm}$ from the sampling orifice. The nano-ESI solutions were loaded into nano-spray emitters with tip orifice diameters of $30 \mu \mathrm{m}$, which were pulled from borosilicate capillaries (1.55 mm o.d., 0.86 mm i.d.) using a P-87 Flaming/Brown micropipette puller (Sutter Instruments, Novato, CA). A stainless steel wire was inserted into the back of the capillary for electrical contact with the analyte solution. The reagent solids, azobenzene in this study, for APCI were placed in a space between the orifice and curtain plate, which helps to increase the vapor pressure of the reagent solids through optimizing the temperature of the atmosphere/vacuum interface by the interface controller. The reagent solutions for ESI were introduced using a Harvard 11 syringe pump (Harvard Apparatus Inc., Holliston, MA) at a flow rate of $1 \mu \mathrm{L} / \mathrm{min}$ into a fused silica capillary (100 $\mu \mathrm{m}$ i.d., $200 \mu \mathrm{m}$ o.d.). Voltages applied to each source were optimized separately and generated independently by a high voltage power supply from the instrument or fast high voltage pulsers (GRX-1.5K-E, Directed Energy Inc., Fort Collins, CO). All the power supplies were triggered from the software so that the high voltages were sequentially switched on/off in each scan. The switching intervals for the oppositely charged high voltage was set to $50 \mathrm{~ms}$ to allow injected ions to be collisionally cooled in the ion trap. The "off" voltages applied to each emitter were $0 \mathrm{~V}$ and the "on" voltages were between \pm 1.2 to 2 $\mathrm{kV}$ for nano-ESI, and $\pm 3 \mathrm{kV}$ for APCI and for ESI. This pulsed source design enables stable ion production from each ion source and allows ions of opposite charge to be generated and injected into the mass spectrometer separately.

All experiments were performed using a prototype version of a Q TRAP mass spectrometer [35] (Applied Biosystems/MDS SCIEX, Concord, Ontario, Canada) and controlled by Daetalyst 3.6 software, a research version of software provided by MDS SCIEX. The $Q$ TRAP electronics were modified to allow superposition of an auxiliary RF signal to the containment lenses of Q2 and Q3 of the LIT [36]. The frequency and amplitude of auxiliary RF signal applied to the containment plates was optimized for each ion/ion reaction experiment. Detailed scan functions for the experiments described herein are provided in the Supplementary Material section (which can be found in the electronic version of this article).

For a typical sequential ion/ion reaction experiment, the high voltage power supply connected to the nanoESI wire was pulsed on to generate analyte ions, which were isolated by Q1 in the RF/DC mode and subsequently injected axially into the Q3 quadrupole array with nitrogen as buffer gas at a pressure of $3.5 \times 10^{-5}$ Torr. The ions were cooled in Q3 for $50 \mathrm{~ms}$, during which time the high voltage on the first emitter was turned off. The ions were sometimes subjected to a second isolation by using Q3 in the RF/DC mode to eliminate fragments resulting from passage through the high-pressure Q2 cell (6-8 mTorr). After the cooling step, the power supply connected to the second ionization source, operated in a polarity opposite to that for previous nano-ESI emitter, was triggered on while the DC potentials applied to the ion path before Q3 were adjusted to allow ions of the other polarity to enter Q3 at relatively low kinetic energies (Q3 DC offset was about $5 \mathrm{~V}$ attractive relative to the Q0 DC offset). During this period, the DC potentials on the Q3 containment lenses, as well as on the Q3 rods themselves, were adjusted to a common value while an auxiliary RF voltage was applied to the exit containment lens. In the subsequent mutual ion polarity storage step, the potential applied to the second ionization source was turned off and the auxiliary RF signals were applied to both containment lenses to store ions axially. After a defined period of mutual storage, a population of product ions with desired polarity was cooled in Q3 for $50 \mathrm{~ms}$ while the auxiliary RF signals were terminated. The other polarity ions were removed by applying an attractive voltage to the containment lenses of Q3. Product ions from the first ion/ion reaction were subjected to another isolation step in the Q3 RF/DC mode if necessary. After another cooling step, the third ionization source was triggered and the second type of reagents ions were injected into Q3 under the same conditions described for the first ion/ion reaction. After another mutual storage period, all the product ions resulting from the second ion/ion reaction were allowed to cool in Q3 for $50 \mathrm{~ms}$ before they were subjected to mass selective axial ejection (MSAE) [37] using a supplementary RF signal at frequency $380 \mathrm{kHz}$. The spectra shown here are typically the averages of 20 to 100 individual scans. Note that although all the ion/ion reactions were conducted in the Q3 LIT, the first step ion/ion reaction in 


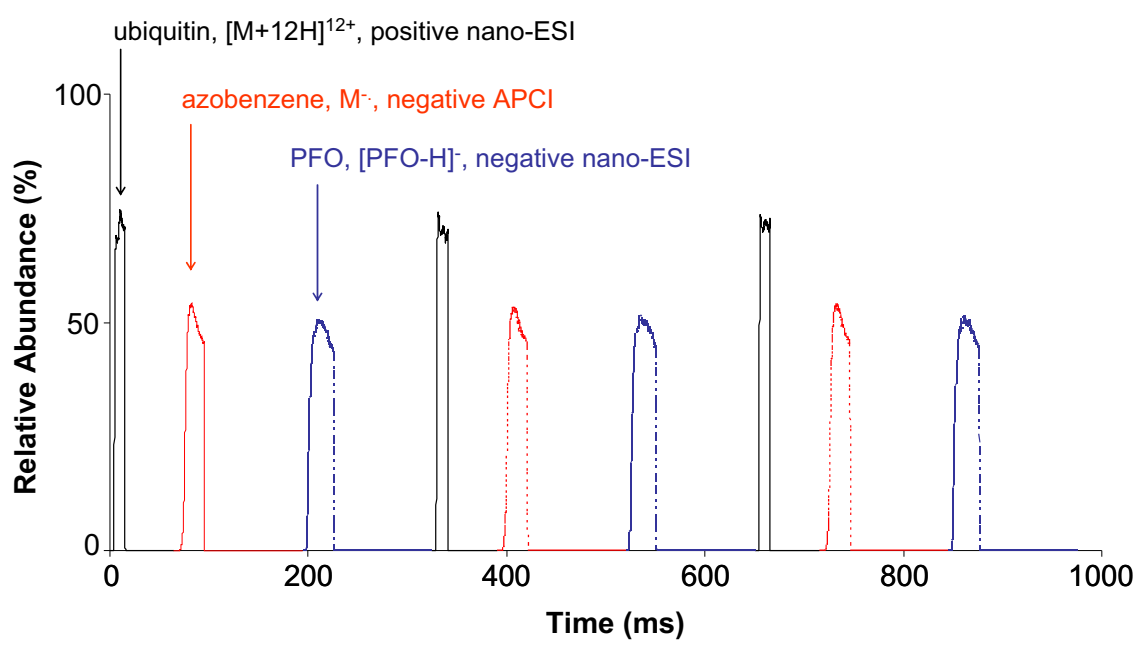

Figure 2. Signals derived from ubiquitin $[\mathrm{M}+12 \mathrm{H}]^{12+}$, formed via positive nano-ESI $(15 \mathrm{~ms}+\mathrm{HV}$ pulse followed by a 50-ms cooling step), radical anion of azobenzene, derived from negative APCI (30 $\mathrm{ms}-\mathrm{HV}$ pulse followed by a 100-ms cooling step), and the deprotonated PFO, formed via negative nano-ESI (30 ms -HV pulse followed by a 100-ms cooling step). In each case, the mass resolving quadrupole was set to pass the ion of interest.

all the studies can also be done in the Q2 LIT, which is a higher pressure cell. Ion/ion reaction rates are usually observed to be one order of magnitude higher in Q2 [33]. However, it is necessary to conduct the second step ion/ion reaction in Q3 if isolation of a product ion of interest is desired from the entire population of product ions resulting from the first step ion/ion reaction. This is because Q2 is a RF-only quadrupole array while Q3 has the capability for RF/DC operation.

\section{Results and Discussion}

\section{Performance Characterization of the Pulsed Triple Ionization Source}

The pulse-to-pulse reproducibility for the sequential pulsed ionization approach described in the experimental section is reflected by the traces in Figure 2, which shows signals from cationic and anionic reactants upon repeated pulsing. The data in Figure 2 reflect good reproducibility of the pulsed triple ionization source, which includes a positive nano-ESI source for ubiquitin (analyte) ion formation, a negative APCI source for azobenzene anion (electron-transfer reagent) formation, and a negative nano-ESI source for the deprotonated perfluoro-1-decanol (proton-transfer reagent) formation. In our experience, the pulse-to-pulse reproducibility for both nano-ESI and APCI sources is similar to that shown in Figure 2.

Good reproducibility for the reagent ions is particularly desirable because it allows for the selection of ion accumulation times that will ensure that the reagent ions are in significant excess over the analyte ion population. When this is the case, reaction rates follow pseudo-first-order kinetics such that the fractional conversion of analyte precursor ions to analyte product ions is independent of the number of analyte ions.
Operating in this way minimizes the need for balancing the numbers of anions and cations.

\section{Charge Increase from a Singly Protonated Peptide to a Doubly Protonated Peptide}

The structural information of gaseous peptide and proteins ions, which are commonly formed either via matrix-assisted laser desorption ionization [38] or ESI [39], is usually obtained from dissociation methods. The preferred dissociation channels for a peptide or protein ion are highly dependent on the charge state of the ion $[40,41]$ regardless of the dissociation methods employed. Two key factors, number and identities of basic amino acid residues, and the ionization mechanism, play roles in determining the initial charge states of a polypeptide when the ions are formed in the gas-phase via ESI. Ion/ion proton-transfer reactions have been employed as a secondary means of manipulating both ion charge and polarity in the gas phase after the ions are formed. Previously, we reported a strategy of increasing the net charge of an ion in the gas phase by using two sequential charge inversion steps [20]. However, such studies have been conducted only with extensively modified instruments, in which two or three distinct electrospray interfaces were incorporated into the design. Here, the pulsed triple ESI source is demonstrated to enable the net charge increase of a peptide ion with minimum instrument modifications using a common ion path and atmosphere/vacuum interface.

Figure 3 summarizes results for an experiment in which two steps of charge inversion were used to form $[\mathrm{M}+2 \mathrm{H}]^{2+}$ ions from $[\mathrm{M}+\mathrm{H}]^{+}$ions using the pulsed triple ESI arrangement for analyte and two types of reagent ions. The first step of the experiment involved 


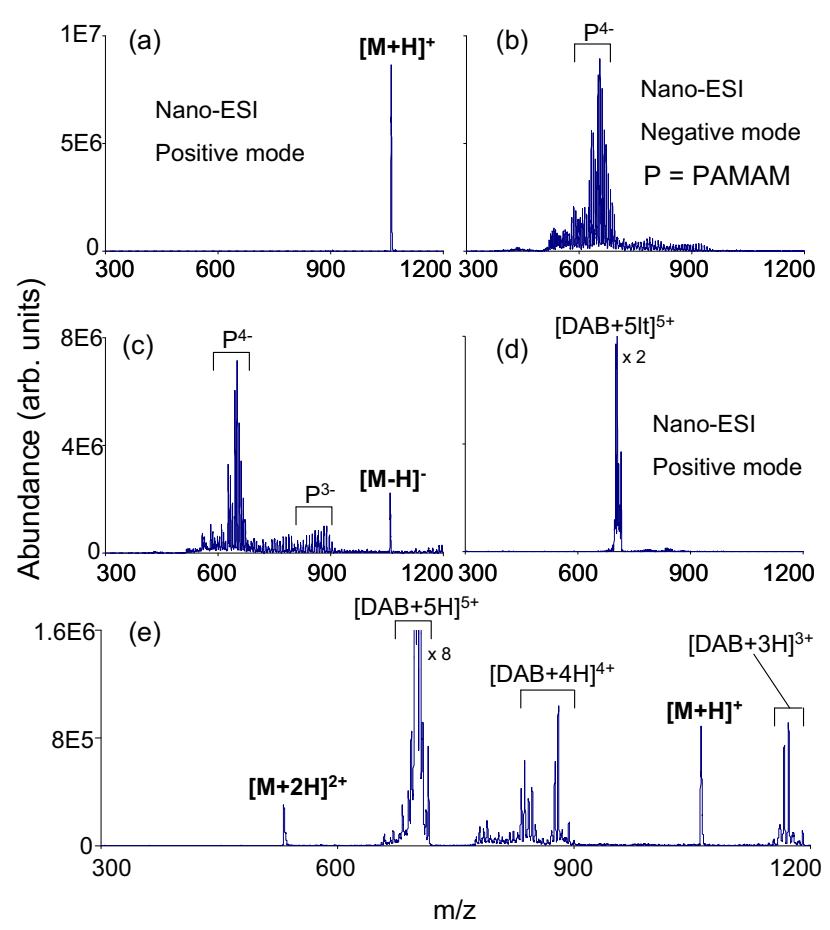

Figure 3. Mass spectra derived from the pulsed triple electrospray source: (a) isolated $[\mathrm{M}+\mathrm{H}]^{+}$ions of bradykinin from positive nano-electrospray; (b) negative electrospray of PAMAM dendrimer generation 1.5; (c) post-ion/ion spectrum acquired in negative ion mode after $500 \mathrm{~ms}$ reaction of ions shown in (a) and (b); (d) isolated $[\mathrm{DAB}+5 \mathrm{H}]^{5+}$ ions of $\mathrm{DAB}$ dendrimer generation 4 from positive electrospray; (e) Post-ion/ion spectrum was acquired in the positive ion mode after a 500-ms reaction between isolated $[\mathrm{M}-\mathrm{H}]^{-}$ions of bradykinin shown in $(\mathrm{c})$ and $[\mathrm{DAB}+5 \mathrm{H}]^{5+}$ ions shown in $(\mathrm{d})$.

the accumulation of bradykinin ions formed via positive nano-ESI in Q3 LIT followed with isolation of the $[\mathrm{M}+\mathrm{H}]^{+}$ion using the Q1 RF/DC mode. The resulting mass spectrum is shown in Figure 3a. The second step is the admission of the multiply charged negative carboxylate-terminated polyamidoamine dendrimer (generation 1.5) (PAMAM) ions to the same linear ion trap. Figure $3 \mathrm{~b}$ shows the mass spectrum of PAMAM ions in the negative mode (no cations present). The main charge state of the PAMAM ions is -4 , denoted as $\mathrm{P}^{4-}$, as well as peaks arising from faulty synthesis products (not labeled in spectrum). Mixtures of sodium ions and protons as counter-ions were present within this charge state of PAMAM ions, which can be expressed as $[\mathrm{P}-(4+\mathrm{m}) \mathrm{Na}+\mathrm{mH}]^{4-}(\mathrm{m}=0-12)$. This mixture of PAMAM ions was allowed to react with [M $+\mathrm{H}]^{+}$ions of bradykinin for $300 \mathrm{~ms}$. Since the PAMAM dendrimer anion signal was spread over a wide variety of anionic species, no ion isolation via Q1 was employed so that the ion/ion reaction rate could be maximized. In the post-ion/ion spectrum of Figure 3c, both residual PAMAM anions and $[\mathrm{M}-\mathrm{H}]^{-}$bradykinin anions were observed. The Bradykinin $[\mathrm{M}-\mathrm{H}]^{-}$ ions were then isolated by using Q3 in the RF/DC mode. Poly(propylenimine) 1,4-diaminobutane (DAB) dendrimer generation 4 cations were formed from positive electrospray with $[\mathrm{DAB}+5 \mathrm{H}]^{5+}$ ions being selected by Q1 for reaction, shown in Figure 3d. Figure 3e shows the ion/ion reaction products after mutual storage for $350 \mathrm{~ms}$ of the isolated bradykinin $[\mathrm{M}-\mathrm{H}]^{-}$ions shown in Figure $3 \mathrm{c}$ and $[\mathrm{DAB}+5 \mathrm{H}]^{5+}$ ions in Figure $3 \mathrm{~d}$. The charge state distribution of the DAB dendrimer ions has shifted to lower charge states as a result of partial neutralization and charge inversion with the bradykinin anions. The charge inversion products are obvious in Figure $3 e$ with $[\mathrm{M}+2 \mathrm{H}]^{2+}$ ions of bradykinin present as well as the corresponding $[\mathrm{M}+\mathrm{H}]^{+}$ions. The relative abundances of $[\mathrm{M}+2 \mathrm{H}]^{2+}$ and $[\mathrm{M}+\mathrm{H}]^{+}$ ions of bradykinin are highly dependent on the identity of the reagent ions, such as charge state, size, and chemical functionalities [20]. In the case of using a pulsed triple electrospray ionization source, the net yield for forming the doubly protonated bradykinin ions from the singly protonated ones is roughly 5 to $10 \%$, which is comparable to our previous reported number by using three distinct electrospray ionization sources in a 3D quadrupole ion trap [20].

\section{Sequential Proton-Transfer Charge Inversion and Electron-Transfer Dissociation of a Phosphopeptide}

Structure interrogation of a protein or peptide with post-translational modifications (PTM) by tandem mass spectrometry via conventional activation methods, such as CID or infrared multiphoton dissociation, often results in difficulties in the identification of the site of modification [42, 43]. Recently, electron capture dissociation (ECD) [44] and electron-transfer dissociation (ETD) [7] of multiply charged cations have shown extensive cleavage of the peptide backbone while preserving PTM groups, such as phosphate and sugar groups. Both ECD and ETD methods require protein and peptide ions to be multiply charged cations. However, the phosphopeptides often give either low or no signal via positive ESI when the phosphopeptide is present with other unmodified peptides. Strong signals, on the other hand, are often generated using negative ESI [22]. So to employ ETD to deduce structural information from many phosphopeptides, it is desirable to be able to convert abundant phosphopeptide anions to multiply charged phosphopeptide cations. In this regard, proton-transfer charge inversion ion/ion chemistry can be readily adapted to serve this purpose. Here we used a pulsed triple ionization source as a tool to characterize the phosphopeptide via sequential charge inversion and electron-transfer dissociation.

The data in Figure 4 illustrate an application of the pulsed triple ionization source in characterizing the phosphopeptide via a two-step ion/ion reaction. In this case, DAB dendrimer generation 4 cations (Figure 4a) are formed from positive nano-ESI with $[\mathrm{DAB}+7 \mathrm{H}]^{7+}$ ions being selected by Q1 for reaction. $[\mathrm{M}-\mathrm{H}]^{-}$ions of LKRApYGL-NH ${ }_{2}$ (Figure $4 \mathrm{~b}$ ) were formed via negative 


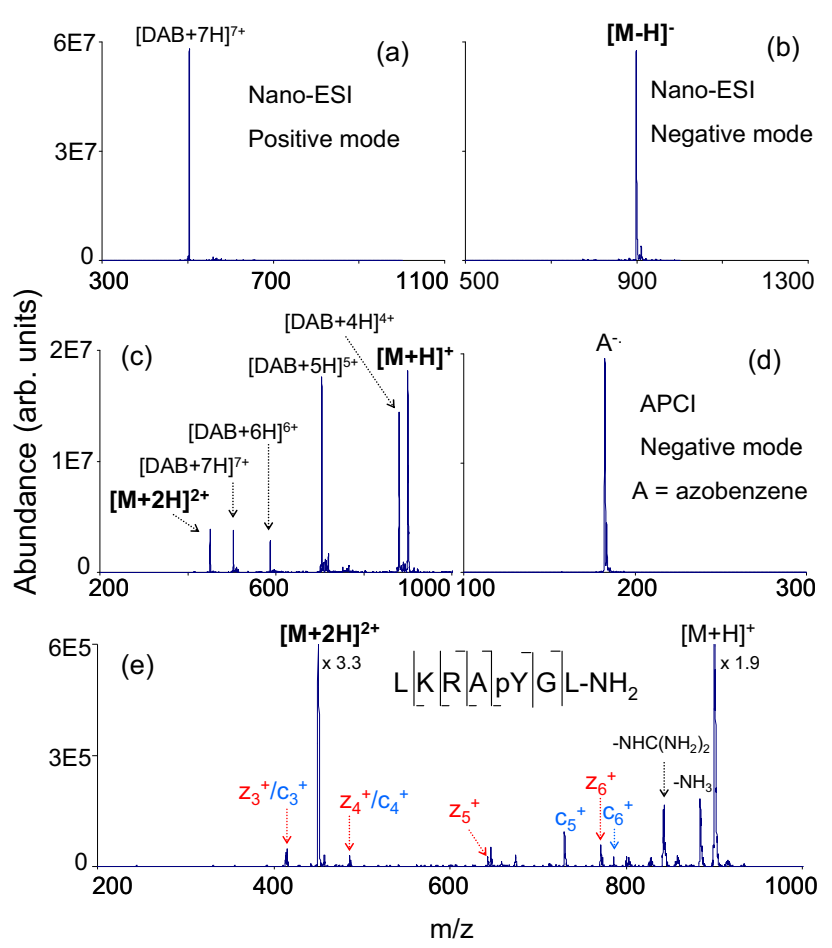

Figure 4. Mass spectra derived from the pulsed triple ionization source: (a) isolated $[\mathrm{DAB}+7 \mathrm{H}]^{7+}$ ions of $\mathrm{DAB}$ dendrimer generation 4 from positive nano-ESI; (b) isolated $[\mathrm{M}-\mathrm{H}]^{-}$ions of peptide LKRApYGL-NH ${ }_{2}$ from negative nano-ESI; (c) post-ion/ion spectrum acquired in positive ion mode after $300 \mathrm{~ms}$ reaction of ions shown in (a) and (b); (d) isolated molecular radical anions of azobenzene from negative APCI; (e) Post-ion/ion spectrum acquired in the positive ion mode after $350 \mathrm{~ms}$ reaction between isolated $[\mathrm{M}+2 \mathrm{H}]^{2+}$ ions of peptide LKRApYGL-NH ${ }_{2}$ shown in (c) and azobenzene molecular radical anions shown in (d).

nano-ESI and isolated using the Q1 in the RF/DC mode. Figure $4 \mathrm{c}$ shows the spectrum resulting from the mutual storage of the ions shown in Figure $4 \mathrm{a}$ and $\mathrm{b}$ for $300 \mathrm{~ms}$. Both singly and doubly protonated species are formed from this phosphopeptide with no triply protonated ions being observed. Considering the relatively small size of this particular phosphopeptide, it may not be expected to form triply protonated ions due to the high electrostatic repulsion from three positive charges. No triply protonated forms of the ion were observed in the positive ion nano-electrospray ionization of this phosphopeptide (data not shown). The charge inverted LKRApYGL- $\mathrm{NH}_{2}[\mathrm{M}+2 \mathrm{H}]^{2+}$ ions were then isolated by using Q3 in the RF/DC mode and subjected to the next step of ion/ion reaction with azobenzene molecular anions (Figure 4d), which were generated through negative APCI and isolated by Q1 in the RF/DC mode when transmitted through the Q1 quadrupole. Azobenzene was chosen as the ETD reagent species because it has been shown previously to lead to relatively high ETD efficiency when reacting with a triply protonated model peptide [45]. Figure 4e shows the electrontransfer ion/ion reaction products after mutual storage of the isolated LKRApYGL-NH ${ }_{2}[\mathrm{M}+\mathrm{H}]^{2+}$ ions via charge inversion shown in Figure $4 \mathrm{c}$ and $[\mathrm{DAB}+7 \mathrm{H}]^{7+}$ ions in Figure $4 \mathrm{~d}$ for $350 \mathrm{~ms}$. Electron-transfer from azobenzene anions to doubly protonated LKRApYGL$\mathrm{NH}_{2}$ gave rise to c- and/or z-type fragments at every inter-residue bond as well as fragments from arginine side-chain loss. The location of the phosphate group is identified by z-type fragment ions $\mathrm{N}$-terminal to tyrosine and c-type fragment ions $\mathrm{C}$-terminal to tyrosine that are 80 mass units higher than the corresponding unmodified peptide. Unlike collisional activation dissociation, the ETD gives no loss of the phosphate group from the c- and z-type ions.

The above approach for the analysis of the phosphopeptide allows ionization of the phosphopeptide in an optimized condition, i.e., nano-ESI in negative mode while utilizing ETD as a structural interrogation tool simultaneously. It is necessary for this approach to use either three distinct ionization sources or a pulsed triple ionization source for independently maximizing the ion signal from each emitter. Compared with the three distinct ionization sources reported previously [22], the advantage of the pulsed triple ionization source described here lies in less required instrument modification work, the use of one common atmospheric/vacuum interface, and the ability to mass select independently each of the reactant species.

\section{Ubiquitin Identification Using the Sequential Electron-Transfer and Proton-Transfer Ion/Ion Reactions}

ECD has been used to directly dissociate whole proteins in a mass spectrometer [46-48]. Most of the resulting product ions are highly charged. The charge states of the product ions can usually be determined directly from the isotope spacings using a Fourier transform ion cyclotron resonance mass spectrometer (FT-ICR-MS) because of its high mass resolving power. However, the use of ECD is essentially restricted to FT-ICR-MS. Recently, a two-step ion/ion reaction approach for top-down protein identification has been demonstrated using a linear ion trap that involves sequential electrontransfer and proton-transfer ion/ion reactions [23]. In this approach, proton-transfer ion/ion reactions are used to simplify the spectrum, make spectral interpretation easier, and concentrate the multiply charged cand z-type fragment ion signals into predominantly one charge state. The strategy is readily implemented with the three-source design whereby the electron-transfer and proton transfer reagents are generated independently from each other and from the analyte ions.

The data in Figure 5 illustrates the identification of ubiquitin by using sequential electron-transfer and proton-transfer ion/ion reactions. Ubiquitin ions were formed via positive nano-ESI with isolation of the [M + $12 \mathrm{H}]^{12+}$ ion using the Q1 RF/DC mode. The resulting mass spectrum is shown in Figure 5a. After ubiquitin ions were cooled for $50 \mathrm{~ms}$ in the Q3 linear ion trap, the radical anions of azobenzene produced via negative 


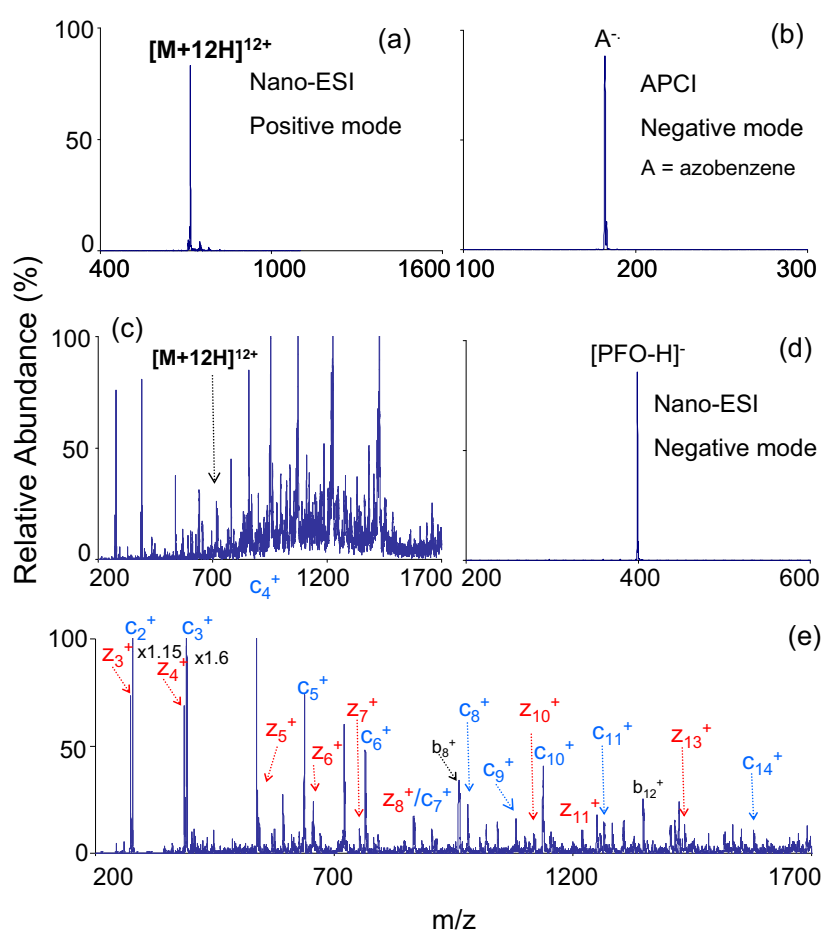

Figure 5. Mass spectra derived from the pulsed triple ionization source: (a) isolated $[\mathrm{M}+12 \mathrm{H}]^{12+}$ ions of bovine ubiquitin from positive nano-ESI; (b) isolated molecular radical anions of azobenzene from negative APCI; (c) post-ion/ion spectrum acquired in the positive ion mode after $300 \mathrm{~ms}$ reaction of ions shown in (a) and $(\mathbf{b}) ;(\mathbf{d})$ isolated $[\mathrm{PFO}-\mathrm{H}]^{-}$ions of perfluoro-1-octanol (PFO) from negative nano-ESI; (E) Post-ion/ion spectrum acquired in positive ion mode after $300 \mathrm{~ms}$ reaction between the population of ions shown in (c) and [PFO-H] $]^{-}$ions shown in (d).

APCI (Figure 5b) were admitted into the same ion trap and were stored for $300 \mathrm{~ms}$. Figure $5 \mathrm{c}$ shows the post-ion/ion spectrum, which is too complicated for direct interpretation. Thus, the entire population of product ions in Figure 5c was subjected to a $300 \mathrm{~ms}$ reaction with the deprotonated perfluoro-1-octanol ions (Figure 5d), which were generated in negative nano-ESI and isolated by Q1. Figure 5e shows the product ion spectrum after the proton-transfer reaction. Most ETD fragment ions (c-and z-type) are singly protonated and can be easily identified.

\section{Conclusions}

A pulsed triple ionization source, typically a nano-ESI emitter for analyte formation and two other emitters, an ESI emitter and an APCI emitter or a second nano-ESI emitter for formation of the two different reagent ions, has been developed and used to produce ions for sequential ion/ion reactions. This work eliminates the need for extensive modifications to the tandem mass spectrometer hardware, while enabling a wide range of two-step ion/ion reactions in an electrodynamic ion trap. The flexibility and analytical utility of the triple ionization source was demonstrated in this paper with charge increase, charge inversion/electron-transfer, and electron-transfer/charge reduction ion/ion reactions. Compared with previously reported dual ESI and ESI/APCI works, this study demonstrated that sequential ion/ion reactions can be readily performed in a straightforward fashion by a triple ionization source. In this regard, this triple ionization source shares the same advantages as the dual ESI and ESI/APCI source, while being able to implement sequential ion/ion reactions in an electrodynamic ion trap using reactants formed via independent ion sources. This triple ionization source is particularly useful for the $\mathrm{MS}^{\mathrm{n}}$ experiments that require more than one type of reagent ion, such as the experiment to increase analyte ion charge via sequential charge inversion reactions.

\section{Acknowledgments}

This work was sponsored by MDS SCIEX, an Industrial Associate of the Department of Chemistry and the National Institutes of Health, Institute of General Medical Sciences under grant GM 45372. The authors acknowledge Dr. James W. Hager and Dr. Frank A. Londry of MDS SCIEX for helpful discussions and Dr. Min Yang of MDS SCIEX for providing custom instrument control software.

\section{References}

1. McLuckey, S. A.; Stephenson, J. L., Jr. Ion/ion chemistry of high-mass multiply charged ions. Mass Spectrom. Rev. 1998, 17, 369-407.

2. Pitteri, S. J.; McLuckey, S. A. Recent developments in the ion/ion chemistry of high-mass multiply charged ions. Mass Spectrom. Rev. 2005, 24, 931-958.

3. Stephenson, J. L., Jr.; McLuckey, S. A. Ion/ion proton transfer reactions for protein mixture analysis. Anal. Chem. 1996, 68, 4026-4032.

4. Stephenson, J. L., Jr.; McLuckey, S. A. Simplification of product ion spectra derived from multiply charged parent ions via ion/ion chemistry. Anal. Chem. 1998, 70, 3533-3544.

5. McLuckey, S. A.; Reid, G. E.; Wells, J. M. Ion parking during ion/ion reactions in electrodynamic ion traps. Anal. Chem. 2002, 74, 336-346.

6. Chrisman, P. A.; Pitteri, S. J.; McLuckey, S. A. Parallel ion parking of protein mixtures. Anal. Chem. 2006, 78, 310-316.

7. Coon, J. J.; Syka, J. E. P.; Schwartz, J. C.; Shabanowitz, J.; Hunt, D. F. Anion dependence in the partitioning between proton and electron transfer in ion/ion reactions. Int. J. Mass Spectrom. 2004, 236, 33-42.

8. Syka, J. E.; Coon, J. J.; Schroeder, M. J.; Shabanowitz, J.; Hunt, D. F. Peptide and protein sequence analysis by electron transfer dissociation mass spectrometry. Proc. Natl. Acad. Sci. U.S.A. 2004, 101, 9528-9533.

9. Chrisman, P. A.; Pitteri, S. J.; Hogan, J. M.; McLuckey, S. A. $\mathrm{SO}_{2}{ }^{-}$ electron transfer ion/ion reactions with disulfide linked polypeptide ions. J. Am. Soc. Mass. Spectrom. 2005, 16, 1020-1030.

10. Hogan, J. M.; Pitteri, S. J.; Chrisman, P. A.; McLuckey, S. A. Complementary structural information from a tryptic N-linked glycopeptide via electron transfer ion/ion reactions and collision-induced dissociation. J. Proteome Res. 2005, 4, 628-632.

11. Pitteri, S. J.; Chrisman, P. A.; Hogan, J. M.; McLuckey, S. A. Electron transfer ion/ion reactions in a three-dimensional quadrupole ion trap: Reactions of doubly and triply protonated peptides with $\mathrm{SO}_{2}{ }^{-}$. Anal. Chem. 2005, 77, 1831-1839.

12. Ogorzalek-Loo, R. R.; Udseth, H. R.; Smith, R. D. A new approach for the study of gas-phase ion-ion reactions using electrospray ionization. J. Am. Soc. Mass Spectrom. 1992, 3, 695-705.

13. Ebeling, D. D.; Westphall, M. S.; Scalf, M.; Smith, L. M. Corona discharge in charge reduction electrospray mass spectrometry. Anal. Chem. 2000, 72, 5158-5161.

14. Frey, B. L.; Lin, Y.; Westphall, M. S.; Smith, L. M. Controlling gas-phase reactions for efficient charge reduction electrospray mass spectrometry of intact proteins. I. Am. Soc. Mass Spectrom. 2005, 16, 1876-1887.

15. Scalf, M.; Westphall, M. S.; Smith, L. M. Charge reduction electrospray mass spectrometry. Anal. Chem. 2000, 72, 52-60.

16. Scalf, M.; Westphall, M. S.; Krause, J.; Kaufman, S. L.; Smith, L. M Controlling charge states of large ions. Science 1999, 283, 194-197.

17. Ogorzalek-Loo, R. R.; Udseth, H. R.; Smith, R. D. Evidence of charge inversion in the reaction of singly charged anions with multiply charged macro-ions. J. Phys. Chem. 1991, 95, 6412-6415.

18. Mather, R. E.; Todd, J. F. J. The Quadrupole Ion Store (Quistor). VII Simultaneous positive-negative ion mass-spectrometry. Int. J. Mass Spectrom. Ion Phys. 1980, 33, 159-165. 
19. McLuckey, S. A.; Glish, G. L.; Van Berkel, G. J. Multiple stages of mass-spectrometry in a quadrupole ion trap mass-spectrometer-prerequisites. Int. J. Mass Spectrom. Ion Processes 1991, 106, 213-235.

20. He, M.; McLuckey, S. A. Two ion/ion charge inversion steps to form a doubly protonated peptide from a singly protonated peptide in the gas phase. I. Am. Chem. Soc. 2003, 125, 7756-7757.

21. He, M.; McLuckey, S. A. Increasing the negative charge of a macroanion in the gas phase via sequential charge inversion reactions. Anal. Chem. 2004, 76, 4189-4192.

22. Gunawardena, H. P.; Emory, J. F.; McLuckey, S. A. Phosphopeptide anion characterization via sequential charge inversion and electrontransfer dissociation. Anal. Chem. 2006, 78, 3788-3793.

23. Coon, J. J.; Ueberheide, B.; Syka, J. E.; Dryhurst, D. D.; Ausio, J.; Shabanowitz, J.; Hunt, D. F. Protein identification using sequential ion/ion reactions and tandem mass spectrometry. Proc. Natl. Acad. Sci. U.S.A. 2005, 102, 9463-9468.

24. Herron, W. J.; Goeringer, D. E. McLuckey, S. A. Gas-Phase Electron Transfer Reactions from Multiply-Charged Anions to Rare Gas Cations. J. Am. Chem. Soc. 1995, 117, 11555-11562.

25. Herron, W. J.; Goeringer, D. E.; McLuckey, S. A. Ion-ion reactions in the gas phase: Proton transfer reactions of protonated pyridine with multiply charged oligonucleotide anions. J. Am. Soc. Mass Spectrom. 1995, 6, 529-532.

26. Payne, A. H.; Glish, G. L. Gas-phase ion/ion interactions between peptides or proteins and iron ions in a quadrupole ion trap. Int. J. Mass Spectrom. 2001, 204

27. Stephenson, J. L., Jr.; McLuckey, S. A. Adaptation of the Paul Trap for study of the reaction of multiply charged cations with singly charged anions. Int. J. Mass Spectrom. Ion Processes 1997, 162, 89-106.

28. Reid, G. E.; Wells, J. M.; Badman, E. R.; McLuckey, S. A. Performance of a quadrupole ion trap mass spectrometer adapted for ion/ion reaction studies. Int. I. Mass Spectrom. 2003, 222, 243-258.

29. Wu, J.; Hager, J. W.; Xia, Y.; Londry, F. A.; McLuckey, S. A. Positive ion transmission mode ion/ion reactions in a hybrid linear ion trap. Anal. Chem. 2004, 76, 5006-5015.

30. Wells, J. M.; Chrisman, P. A.; McLuckey, S. A. "Dueling" ESI: Instrumentation to study ion/ion reactions of electrospray-generated cations and anions. J. Am. Soc. Mass Spectrom. 2002, 13, 614-622.

31. Badman, E. R.; Chrisman, P. A.; McLuckey, S. A. A quadrupole ion trap mass spectrometer with three independent ion sources for the study of gas-phase ion/ion reactions. Anal. Chem. 2002, 74, 6237-6243.

32. Xia, Y.; Liang, X.; McLuckey, S. A. Sonic spray as a dual polarity ion source for ion/ion reactions. Anal. Chem. 2005, 77, 3683-3689.

33. Xia, Y.; Liang, X.; McLuckey, S. A. Pulsed dual electrospray ionization for ion/ion reactions. J. Am. Soc. Mass Spectrom. 2005, 16, 1750-1756.

34. Liang, X.; Xia, Y.; McLuckey, S. A. Alternately pulsed nanoelectrospray ionization/atmospheric pressure chemical ionization for ion/ion reactions in an electrodynamic ion trap. Anal. Chem. 2006, 78, 3208-3212.
35. Hager, J. W. A new linear ion trap mass spectrometer. Rapid Commun. Mass Spectrom. 2002, 16, 512-526.

36. Xia, Y.; Wu, J.; McLuckey, S. A.; Londry, F. A.; Hager, J. W. Mutual storage mode ion/ion reactions in a hybrid linear ion trap. J. Am. Soc. Mass Spectrom. 2005, 16, 71-81.

37. Londry, F. A.; Hager, J. W. Mass selective axial ion ejection from a linea quadrupole ion trap. J. Am. Soc. Mass Spectrom. 2003, 14, 1130-1147.

38. Karas, M.; Hillenkamp, F. Laser desorption ionization of proteins with molecular masses exceeding 10,000 daltons. Anal. Chem. 1988, 60, 2299-2301.

39. Fenn, J. B.; Mann, M.; Meng, C. K.; Wong, S. F.; Whitehouse, C. M Electrospray ionization for mass spectrometry of large biomolecules. Science 1989, 246, 64-71.

40. Dongré, A. R.; Jones, J. L.; Somogyi, Á.; Wysocki, V. H. Influence of peptide composition, gas-phase basicity, and chemical modification on fragmentation efficiency: Evidence for the mobile proton model. J. Am. Chem. Soc. 1996, 118, 8365-8374.

41. Reid, G. E.; Wu, J.; Chrisman, P. A.; Wells, J. M.; McLuckey, S. A Charge-state-dependent sequence analysis of protonated ubiquitin ions via ion trap tandem mass spectrometry. Anal. Chem. 2001, 73, 32743281.

42. Flora, J. W.; Muddiman, D. C. Selective, sensitive, and rapid phosphopeptide identification in enzymatic digests using ESI-FTICR-MS with infrared multiphoton dissociation. Anal. Chem. 2001, 73, 3305-3311.

43. Annan, R. S.; Carr, S. A. Phosphopeptide analysis by matrix-assisted laser desorption time-of-flight mass spectrometry. Anal. Chem. 1996, 68, 3413-3421.

44. Stone, D. H.; Hemling, M. E.; Carr, S. A.; Horn, D. M.; Lindh, I.; McLafferty, F. W. Phosphopeptide/phosphoprotein mapping by electron capture dissociation mass spectrometry. Anal. Chem. 2001, 73, 19-22.

45. Gunawardena, H. P.; He, M.; Chrisman, P. A.; Pitteri, S. J.; Hogan, J. M. Hodges, B. D.; McLuckey, S. A. Electron transfer versus proton transfer in gas-phase ion/ion reactions of polyprotonated peptides. J. Am. Chem. Soc. 2005, 127, 12627-12639.

46. Ge, Y.; El-Naggar, M.; Sze, S. K.; Oh, H. B.; Begley, T. P.; McLafferty, F. W.; Boshoff, H.; Barry, C. E. III. Top down characterization of secreted proteins from Mycobacterium tuberculosis by electron capture dissociation mass spectrometry. J. Am. Soc. Mass Spectrom. 2003, 14, 253-261.

47. Ge, Y.; Lawhorn, B. G.; ElNaggar, M.; Strauss, E.; Park, J. H.; Begley T. P.; McLafferty, F. W. Top down characterization of larger proteins (45 $\mathrm{kDa}$ ) by electron capture dissociation mass spectrometry. J. Am. Chem. Soc. 2002, 124, 672-678.

48. Sze, S. K.; Ge, Y.; Oh, H.; McLafferty, F. W. Top-down mass spectrometry of a $29-\mathrm{kDa}$ protein for characterization of any posttranslationa modification to within one residue. Proc. Natl. Acad. Sci. U.S.A. 2002, 99, 1774-1779. 